Volume 70, Number 1, Pages 320-330 (2021)

DOI: $10.31801 /$ cfsuasmas.729550

ISSN 1303-5991 E-ISSN 2618-6470

Received by the editors: April 29, 2020; Accepted:January 5, 2021

\title{
A METRIC FORMULA ON A QUOTIENT SPACE WHICH IS RELATED TO THE SEQUENCE SPACE $\Sigma_{2}$
}

\author{
Mustafa SALTAN and Nisa ASLAN \\ Department of Mathematics, Eskişehir Technical University, 26470, Eskişehir, TURKEY
}

\begin{abstract}
In this paper, we first define an equivalence relation on the sequence space $\Sigma_{2}$. Then we equip the quotient set $\Sigma_{2} / \sim$ with a metric $d_{1}$. We also determine an isometry map between the metric spaces $\left(\Sigma_{2} / \sim, d_{1}\right)$ and $\left([0,1], d_{\text {eucl }}\right)$. Finally, we investigate the symmetry conditions with respect to some points on the metric space $\left(\Sigma_{2} / \sim, d_{1}\right)$ and we compare truncation errors for the computations which is obtained by the metrics $d_{\text {eucl }}$ and $d_{1}$.
\end{abstract}

\section{INTRODUCTION}

Let $\Sigma_{n}$ denote the set of all infinite sequences of 0's, 1's, $\ldots, n-1$ 's. That is,

$$
\Sigma_{n}=\left\{s_{1} s_{2} s_{3} \ldots \mid s_{i} \in\{0,1, \ldots, n-1\}\right\}
$$

(see [1,2,8]). This set is called the sequence space on the $n$ symbols $0,1, \ldots, n-1$. $\left(\Sigma_{n}, d\right)$ is the code space on $n$ symbols with the metric

$$
d(s, t)=\sum_{i=1}^{\infty} \frac{\left|s_{i}-t_{i}\right|}{n^{i}} \text { for all } s, t \in \Sigma_{n} .
$$

A semi-metric on $\Sigma_{n}$ is also defined as follows:

$$
d^{\prime}(s, t)=\left|\sum_{i=1}^{\infty} \frac{s_{i}-t_{i}}{n^{i}}\right| \text { for all } s, t \in \Sigma_{n} .
$$

It is well-known that there is the surjective mapping $h$ between $\Sigma_{2}$ and $[0,1]$ such that

$$
h: \Sigma_{2} \rightarrow[0,1], h\left(s_{1} s_{2} \ldots s_{n} \ldots\right)=\frac{s_{1}}{2}+\frac{s_{2}}{2^{2}}+\ldots+\frac{s_{n}}{2^{n}}+\ldots
$$

2020 Mathematics Subject Classification. 28A80, 51F99.

Keywords and phrases. Metric spaces, symbolic dynamics, isometry, symmetry, truncation errors.

\mustafasaltan@eskisehir.edu.tr; nisakucuk@eskisehir.edu.tr-Corresponding author

(D) 0000-0002-3252-3012; 0000-0002-2103-0511. 
But, the mapping $h$ is not injective. To exemplify, let us consider $01111 \ldots$ and $1000 \ldots$ of $\Sigma_{2}$. It is clear that

$$
h(01111 \ldots)=h(1000 \ldots)=\frac{1}{2} .
$$

Generally, any rational number $\frac{p}{2^{k}} \in[0,1](p, k$ are integers) has exactly two preimages $s^{\prime}, s^{\prime \prime}$ under $h$; they have forms $s^{\prime}=s_{1} s_{2} \ldots s_{n} \alpha \bar{\beta}$ and $s^{\prime \prime}=s_{1} s_{2} \ldots s_{n} \beta \bar{\alpha}$ for some $n \in \mathbb{N}, s_{i}, \alpha, \beta \in\{0,1\}$ and $\bar{a}$ stands for the sequence in $\Sigma_{2}$ with all the items equal to $a$ where $a \in\{0,1\}$. To obtain an injective mapping, an equivalence relation $\sim$ on $\Sigma_{2}$ is defined by

$$
\begin{aligned}
s^{\prime} \sim s^{\prime \prime} \Leftrightarrow \quad \begin{array}{l}
s^{\prime} \\
s^{\prime}
\end{array}=s^{\prime \prime} \text { or there are } s_{i}, \alpha, \beta \in\{0,1\} \text { such that } \\
s_{2} \ldots s_{n} \alpha \bar{\beta}, s^{\prime \prime}=s_{1} s_{2} \ldots s_{n} \beta \bar{\alpha} \text { for an integer } n .
\end{aligned}
$$

Therefore, the mapping $h^{\prime}$ defined as

$$
\begin{gathered}
h^{\prime}: \Sigma_{2} / \sim \rightarrow[0,1] \\
h^{\prime}\left(s_{1} s_{2} \ldots s_{n} \ldots\right)=\frac{s_{1}}{2}+\frac{s_{2}}{2^{2}}+\ldots+\frac{s_{n}}{2^{n}}+\ldots
\end{gathered}
$$

is bijective.

From now on, one can identify each element of $\Sigma_{2} / \sim$ with an appropriate element of $[0,1]$. That is, $\Sigma_{2} / \sim$ can be considered as the code set of the points on $[0,1]$. Note that, $d$ is not well defined on $\Sigma_{2} / \sim$. For example, for the elements $s=00 \overline{1}$, $s^{\prime}=0 \overline{1}$ and $s^{\prime \prime}=1 \overline{0}\left(s^{\prime} \sim s^{\prime \prime}\right)$ of $\Sigma_{2} / \sim$, we compute $d\left(s, s^{\prime}\right) \neq d\left(s, s^{\prime \prime}\right)$. Therefore, $d$ is not a metric on $\Sigma_{2} / \sim$, but $\left(\Sigma_{2} / \sim, d^{\prime}\right)$ is a metric space. Obviously, the metric spaces $\left(\Sigma_{2} / \sim, d^{\prime}\right)$ and $\left([0,1], d_{\text {eucl }}\right)$ are isometric since $h^{\prime}$ is an isometry. On the other hand, different metrics can be defined on the same set $X$. The interesting question is whether these metrics are equivalent or not. Because equivalent metrics on the same set $X$ generate the same topology (see [3]). It is a remarkable problem, how to define a metric $d_{1}$, which is formulated in a different way from the metric $d^{\prime}$ given in $(2)$ on $\Sigma_{2} / \sim$ such that $\left(\Sigma_{2} / \sim, d_{1}\right)$ and $\left([0,1], d_{\text {eucl }}\right)$ are isometric.

Any set can be expressed as a quotient space which is related to a sequence space. Using the metrics defined on code sets, many geometric features of the corresponding space can be proved. Moreover, unusual metric formulas can be obtained on different quotient spaces. For example, the Sierpinski Gasket which is one of the fundamental models of fractals can be defined as the quotient space $\Sigma_{3} / \sim^{\prime}$ where

$$
\begin{aligned}
c^{\prime} \sim^{\prime} c^{\prime \prime} \Leftrightarrow \quad c^{\prime} & =c^{\prime \prime} \text { or there are } c_{i}, \alpha, \beta \in\{0,1,2\} \text { such that } \\
c^{\prime} & =c_{1} c_{2} \ldots c_{n} \alpha \bar{\beta}, c^{\prime \prime}=c_{1} c_{2} \ldots c_{n} \beta \bar{\alpha} \text { for an integer } n .
\end{aligned}
$$

(see Figure 1).

In [6], the geodesic metric on the code set of the Sierpinski Gasket is defined as follows: 
Definition 1. Let $a_{1} a_{2} \ldots a_{k-1} a_{k} a_{k+1} \ldots$ and $b_{1} b_{2} \ldots b_{k-1} b_{k} b_{k+1} \ldots$ be two representations of the points $a$ and $b$ respectively on the Sierpinski Gasket such that $a_{i}=b_{i}$ for $i=1,2, \ldots, k-1$ and $a_{k} \neq b_{k}$. The distance $d_{g e o}(a, b)$ between $a$ and $b$ is determined with the following formula:

$$
d_{\text {geo }}(a, b)=\min \left\{\sum_{i=k+1}^{\infty} \frac{\alpha_{i}+\beta_{i}}{2^{i}}, \frac{1}{2^{k}}+\sum_{i=k+1}^{\infty} \frac{\gamma_{i}+\delta_{i}}{2^{i}}\right\}
$$

where

$$
\begin{aligned}
\alpha_{i}=\left\{\begin{array}{ll}
0, & a_{i}=b_{k} \\
1, & a_{i} \neq b_{k}
\end{array},\right. & \beta_{i}= \begin{cases}0, & b_{i}=a_{k} \\
1, & b_{i} \neq a_{k}\end{cases} \\
\gamma_{i}=\left\{\begin{array}{ll}
0, & a_{i} \neq a_{k} \text { and } a_{i} \neq b_{k} \\
1, & \text { otherwise }
\end{array},\right. & \delta_{i}=\left\{\begin{array}{ll}
0, & b_{i} \neq b_{k} \\
1, & \text { otherwise } b_{i} \neq a_{k}
\end{array} .\right.
\end{aligned}
$$

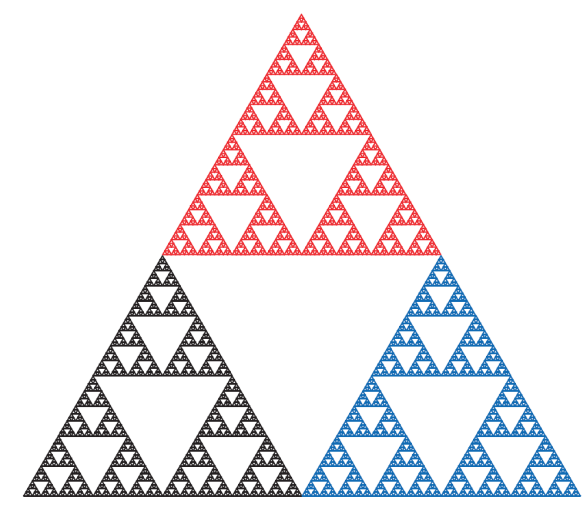

Figure 1. The Sierpinski Gasket

This metric formula describes the shortest distance between any two points on the code sets of the Sierpinski Gasket. Thanks to the metric formula $d_{g e o}$, many different and interesting properties of the Sierpinski Gasket can be proved (for details see 6 6 7 ).

As seen in (6), this metric is actually expressed on the quotient space $\Sigma_{3} / \sim^{\prime}$. In a similar way, a metric on the quotient space $\Sigma_{2} / \sim$ can be defined. Note that, although relations $\sim$ and $\sim^{\prime}$ defined in (3) and (5) are quite similar, the corresponding spaces are different.

In this paper, to define the metric $d_{1}$ we take into account the construction of the intrinsic metric on the Sierpinski Gasket (for details see proof of the Theorem 1 in the paper [5]). We use the quotient space $\Sigma_{2} / \sim$ given in (3) instead of the quotient space $\Sigma_{3} / \sim^{\prime}$ given in (5) which is the code set of the Sierpinski Gasket. We also take the first sum, the same $\alpha_{i}$ and $\beta_{i}$ given in the metric formula (6). Thus we obtain the metric space $\left(\Sigma_{2} / \sim, d_{1}\right)$ and show that this metric space is isometric to 
the metric space $\left([0,1], d_{\text {eucl }}\right)$ in Proposition 1 . Since $d_{1}$ has a different construction than the metric $d_{\text {eucl }}$, we compare some properties of these two metrics. So, we get some interesting results in Proposition 6 and Proposition 8.

\section{A DIFFERENTLY FORMULATED METRIC ON THE QUOTIENT $\operatorname{SET} \Sigma_{2} / \sim$}

We first define a distance on the quotient set $\Sigma_{2} / \sim$ and then we show that this distance function is a metric on $\Sigma_{2} / \sim$.

Suppose that $a=a_{1} a_{2} \ldots a_{k-1} a_{k} a_{k+1} \ldots$ and $b=b_{1} b_{2} \ldots b_{k-1} b_{k} b_{k+1} \ldots$ are two elements of $\Sigma_{2} / \sim$. If $a_{i}=b_{i}$ for all $i=1,2,3, \ldots$, then $d_{1}(a, b)=0$. If $a_{i}$ does not equal to $b_{i}$ for any $i=1,2,3, \ldots$, then there is at least one $k$ such that $a_{k} \neq b_{k} . k$ is chosen as the smallest index $i=1,2, \ldots$ such that $a_{i} \neq b_{i}$. That is, let $a_{i}=b_{i}$ for $i=1,2, \ldots, k-1$ and $a_{k} \neq b_{k}$. We define the distance $d_{1}(a, b)$ between $a$ and $b$ as

$$
d_{1}(a, b)=\sum_{i=k+1}^{\infty} \frac{\alpha_{i}+\beta_{i}}{2^{i}}
$$

where

$$
\alpha_{i}=\left\{\begin{array}{ll}
0 & \text { if } a_{i}=b_{k} \\
1 & \text { if } a_{i} \neq b_{k}
\end{array} \quad, \quad \beta_{i}=\left\{\begin{array}{ll}
0 & \text { if } b_{i}=a_{k} \\
1 & \text { if } b_{i} \neq a_{k}
\end{array} .\right.\right.
$$

Since there are many stages to prove that $\left(\Sigma_{2} / \sim, d_{1}\right)$ is a metric space, we give it as a result of the following proposition:

Proposition 2. $d_{\text {eucl }}\left(h^{\prime}(a), h^{\prime}(b)\right)=d_{1}(a, b)$ for all $a, b \in \Sigma_{2} / \sim$. Thus, $\left(\Sigma_{2} / \sim, d_{1}\right)$ is a metric space.

Proof. Let us first consider the bijective mapping $h^{\prime}$ defined in (4) as follows:

$$
\begin{gathered}
h^{\prime}: \Sigma_{2} / \sim \rightarrow[0,1] \\
h^{\prime}\left(s_{1} s_{2} \ldots s_{n} \ldots\right)=\frac{s_{1}}{2}+\frac{s_{2}}{2^{2}}+\ldots+\frac{s_{n}}{2^{n}}+\ldots
\end{gathered}
$$

We now show that

$$
d_{\text {eucl }}\left(h^{\prime}(a), h^{\prime}(b)\right)=d_{1}(a, b)
$$

for all $a, b \in \Sigma_{2} / \sim$. Suppose that

$$
\begin{aligned}
& a=a_{1} \quad a_{2} \quad \ldots \quad a_{k-1} \quad a_{k} \quad a_{k+1} \quad a_{k+2} \quad \ldots \\
& b=b_{1} \quad b_{2} \quad \ldots \quad b_{k-1} \quad b_{k} \quad b_{k+1} \quad b_{k+2} \ldots
\end{aligned}
$$

where $a_{i}=b_{i}$ for $i=1,2, \ldots, k-1$ and $a_{k} \neq b_{k}$. So, we have either $a_{k}>b_{k}$ or $a_{k}<b_{k}$. Assume that $a_{k}<b_{k}$ (that is, chosen as $a_{k}=0$ and $b_{k}=1$ ). Then, 
$\left|a_{i+1}-b_{k}\right|=b_{k}-a_{i+1}$ and $\left|b_{i+1}-a_{k}\right|=b_{i+1}-a_{k}$ for $i=k, k+1, k+2, \ldots$. The other case is done similarly. Observe that

$$
\begin{aligned}
d_{1}(a, b) & =\sum_{i=k+1}^{\infty} \frac{\alpha_{i}+\beta_{i}}{2^{i}} \\
& =\frac{\left|a_{k+1}-b_{k}\right|+\left|b_{k+1}-a_{k}\right|}{2^{k+1}}+\frac{\left|a_{k+2}-b_{k}\right|+\left|b_{k+2}-a_{k}\right|}{2^{k+2}}+\cdots \\
& =\frac{\left(b_{k}-a_{k+1}\right)+\left(b_{k+1}-a_{k}\right)}{2^{k+1}}+\frac{\left(b_{k}-a_{k+2}\right)+\left(b_{k+2}-a_{k}\right)}{2^{k+2}}+\cdots \\
& =\frac{\left(b_{k}-a_{k}\right)+\left(b_{k+1}-a_{k+1}\right)}{2^{k+1}}+\frac{\left(b_{k}-a_{k}\right)+\left(b_{k+2}-a_{k+2}\right)}{2^{k+2}}+\cdots \\
& =\left(b_{k}-a_{k}\right)\left(\frac{1}{2^{k+1}}+\frac{1}{2^{k+2}}+\cdots\right)+\frac{\left(b_{k+1}-a_{k+1}\right)}{2^{k+1}}+\frac{\left(b_{k+2}-a_{k+2}\right)}{2^{k+2}}+\cdots \\
& =\frac{\left(b_{k}-a_{k}\right)}{2^{k}}+\frac{\left(b_{k+1}-a_{k+1}\right)}{2^{k+1}}+\frac{\left(b_{k+2}-a_{k+2}\right)}{2^{k+2}}+\cdots \\
& =\left(\frac{b_{k}}{2^{k}}+\frac{b_{k+1}}{2^{k+1}}+\frac{b_{k+2}}{2^{k+2}}+\cdots\right)-\left(\frac{a_{k}}{2^{k}}+\frac{a_{k+1}}{2^{k+1}}+\frac{a_{k+2}}{2^{k+2}}+\cdots\right) \\
& =h^{\prime}(b)-h^{\prime}(a) \\
& =\left|h^{\prime}(a)-h^{\prime}(b)\right| \\
& =d_{\text {eucl }}\left(h^{\prime}(a), h^{\prime}(b)\right)
\end{aligned}
$$

So, $\left(\Sigma_{2} / \sim, d_{1}\right)$ is a metric space and it is also isometric to the metric space $\left([0,1], d_{\text {eucl }}\right)$. This concludes the proof.

Observe that if we define the mapping $h^{\prime \prime}$ as below, we have the following result:

$$
\begin{aligned}
& h^{\prime \prime}:\left(\Sigma_{2} / \sim, d_{2}\right) \rightarrow\left([m, n], d_{\text {eucl }}\right) \\
& h^{\prime \prime}\left(s_{1} s_{2} \ldots s_{n} \ldots\right)=m+(n-m)\left(\frac{s_{1}}{2}+\frac{s_{2}}{2^{2}}+\ldots+\frac{s_{n}}{2^{n}}+\ldots\right)
\end{aligned}
$$

where

$$
d_{2}(a, b)=\sum_{i=k+1}^{\infty} \frac{\alpha_{i}^{\prime}+\beta_{i}^{\prime}}{2^{i}}
$$

such that

$$
\alpha_{i}^{\prime}=\left\{\begin{array}{ll}
0 & \text { if } a_{i}=b_{k} \\
n-m & \text { if } a_{i} \neq b_{k}
\end{array} \quad, \quad \beta_{i}^{\prime}=\left\{\begin{array}{ll}
0 & \text { if } b_{i}=a_{k} \\
n-m & \text { if } b_{i} \neq a_{k}
\end{array} \quad \text { where } i \geq k+1 .\right.\right.
$$

Corollary 3. The metric space $\left(\Sigma_{2} / \sim, d_{2}\right)$ is isometric to the metric space $\left([m, n], d_{\text {eucl }}\right)$ for any $m, n \in \mathbb{R}$ and $n>m$. 
Remark 4. One can also use the metric $d_{2}$ defined on $\Sigma_{2} / \sim$ instead of the metric $d_{\text {eucl }}$ defined on $[m, n]$ because of Corollary (3).

Using the metric $d_{1}$, we can make some calculations easier than using Euclidean metric. To better understand the importance of this metric, we will give the following example:

Example 5. Consider the points $a=\overline{010100}$ and $b=\overline{101011}$ of $\Sigma_{2} / \sim$. Let us first compute $d_{1}(a, b)$ and then let us find the point c of $\Sigma_{2} / \sim$ such that $d_{1}(a, b)=d_{1}(\overline{0}, c)$.

$$
\begin{aligned}
d_{1}(a, b)= & \sum_{i=k+1}^{\infty} \frac{\alpha_{i}+\beta_{i}}{2^{i}} \\
= & \left(\frac{0+0}{2^{2}}+\frac{1+1}{2^{3}}+\frac{0+0}{2^{4}}+\frac{1+1}{2^{5}}+\frac{1+1}{2^{6}}+\frac{1+1}{2^{7}}\right)+ \\
& \left(\frac{0+0}{2^{8}}+\frac{1+1}{2^{9}}+\frac{0+0}{2^{10}}+\frac{1+1}{2^{11}}+\frac{1+1}{2^{12}}+\frac{1+1}{2^{13}}\right)+\cdots \\
= & \left(\frac{1}{2^{2}}+\frac{1}{2^{4}}+\frac{1}{2^{5}}+\frac{1}{2^{6}}\right)+\left(\frac{1}{2^{8}}+\frac{1}{2^{10}}+\frac{1}{2^{11}}+\frac{1}{2^{12}}\right)+\cdots \\
= & \frac{1}{2^{2}}\left(1+\frac{1}{2^{6}}+\frac{1}{2^{12}}+\cdots\right)+\frac{1}{2^{4}}\left(1+\frac{1}{2^{6}}+\frac{1}{2^{12}}+\cdots\right) \\
& +\frac{1}{2^{5}}\left(1+\frac{1}{2^{6}}+\frac{1}{2^{12}}+\cdots\right)+\frac{1}{2^{6}}\left(1+\frac{1}{2^{6}}+\frac{1}{2^{12}}+\cdots\right) \\
= & \frac{64}{63}\left(\frac{1}{2^{2}}+\frac{1}{2^{4}}+\frac{1}{2^{5}}+\frac{1}{2^{6}}\right) \\
= & \frac{64}{63} \cdot \frac{23}{64}=\frac{23}{63}
\end{aligned}
$$

Note that the calculation above gives us the point $c$ of $\Sigma_{2} / \sim$ such that $d_{1}(a, b)=$ $d_{1}(\overline{0}, c)$. That is, the point $c$ equals to

$$
\left(\frac{0}{2^{1}}+\frac{1}{2^{2}}+\frac{0}{2^{3}}+\frac{1}{2^{4}}+\frac{1}{2^{5}}+\frac{1}{2^{6}}\right)+\left(\frac{0}{2^{7}}+\frac{1}{2^{8}}+\frac{0}{2^{9}}+\frac{1}{2^{10}}+\frac{1}{2^{11}}+\frac{1}{2^{12}}\right)+\cdots
$$

or equals to $\overline{010111}$ as an element of $\Sigma_{2} / \sim$. If we try to calculate this distance and to find the point $c$ with the Euclidean metric, then we will have to deal with a bit more processing. Because we do not deal with subtraction and absolute value in calculations made with the metric $d_{1}$. Moreover, we directly obtain this distance as a binary number.

In the following proposition, we emphasize a geometrical property of the metric $d_{1}$ different form the metric $d_{\text {eucl }}$. 
Proposition 6. Let $a=a_{1} a_{2} \ldots a_{k-1} a_{k} a_{k+1} \ldots$ and $b=b_{1} b_{2} \ldots b_{k-1} b_{k} b_{k+1} \ldots$ be arbitrary elements of $\Sigma_{2} / \sim$. If $a_{1} \neq b_{1}$ and $\alpha_{i}=\beta_{i}$ for all $i=2,3, \ldots$, then these points are symmetric with respect to the point $0 \overline{1}$ (or equivalently $1 \overline{0}$ ).

Proof. We must show that

$$
d_{1}\left(a_{1} a_{2} \ldots a_{k-1} a_{k} a_{k+1} \ldots, 0 \overline{1}\right)=d_{1}\left(b_{1} b_{2} \ldots b_{k-1} b_{k} b_{k+1} \ldots, 0 \overline{1}\right) .
$$

Then

$$
d_{1}\left(a_{1} a_{2} \ldots a_{k-1} a_{k} a_{k+1} \ldots, 0 \overline{1}\right)=\sum_{i=k+1}^{\infty} \frac{\alpha_{i}^{\prime}+\beta_{i}^{\prime}}{2^{i}}
$$

and

$$
d_{1}\left(b_{1} b_{2} \ldots b_{k-1} b_{k} b_{k+1} \ldots, 0 \overline{1}\right)=\sum_{i=k+1}^{\infty} \frac{\alpha_{i}^{\prime \prime}+\beta_{i}^{\prime \prime}}{2^{i}}
$$

where $\alpha_{i}^{\prime}, \alpha_{i}^{\prime \prime}, \beta_{i}^{\prime}$ and $\beta_{i}^{\prime \prime}$ are determined in accordance with (7). Since $a_{1} \neq b_{1}$, we have

$$
\alpha_{i}=\left\{\begin{array}{ll}
0 & \text { if } a_{i}=b_{1} \\
1 & \text { if } a_{i} \neq b_{1}
\end{array}, \beta_{i}= \begin{cases}0 & \text { if } b_{i}=a_{1} \\
1 & \text { if } b_{i} \neq a_{1}\end{cases}\right.
$$

and $\alpha_{i}=\beta_{i}$ for all $i=2,3, \ldots$. In both cases, we get $b_{i} \neq a_{i}$ for all $i=2,3, \ldots$ because $a_{1}=0, b_{1}=1$ or $a_{1}=1, b_{1}=0$. Let us compute and compare the two distances between $a_{1} a_{2} \ldots a_{k-1} a_{k} a_{k+1} \ldots$ and $0 \overline{1}$ and the distance between $b_{1} b_{2} \ldots b_{k-1} b_{k} b_{k+1} \ldots$ and $0 \overline{1}$. Without loss of generality, suppose that $a_{1}=0$ and $b_{1}=1$. For all $i=2,3, \ldots$ if $a_{i}=1$ then $b_{i}=0$ and $a_{i}=1$ for all $i>1$ yields

$$
d_{1}\left(a_{1} a_{2} \ldots a_{k-1} a_{k} a_{k+1} \ldots, 0 \overline{1}\right)=d_{1}\left(b_{1} b_{2} \ldots b_{k-1} b_{k} b_{k+1} \ldots, 0 \overline{1}\right)=0 .
$$

Otherwise, there is at least one $s$ such that $a_{s}=0$ and $a_{i}=1$ for $i=2,3, \ldots, s-1$ and then we have $b_{s}=1$ and $b_{i}=0$ for $i=2,3, \ldots, s-1$. We obtain $\alpha_{i}^{\prime \prime}=0$ for all $i=2,3, \ldots, s-1$ and $\alpha_{s}^{\prime \prime}=1$, and also $\beta_{i}^{\prime}=1$ for all $i=s+1, s+2, s+3, \ldots$ and $\beta_{i}^{\prime \prime}=0$ for all $i=2,3,4, \ldots$. Furthermore, for any $t \in\{s+1, s+2, s+3, \ldots\}$, if $a_{t}=1$, then we get $b_{t}=0$ and thus $\alpha_{t}^{\prime}=0$ and $\alpha_{t}^{\prime \prime}=0$. Similarly, for any $t \in\{s+1, s+2, s+3, \ldots\}$, if $a_{t}=0$, then we obtain $b_{t}=1$ and thus $\alpha_{t}^{\prime}=1$ and $\alpha_{t}^{\prime \prime}=1$. Therefore

$$
\begin{aligned}
d_{1}\left(a_{1} a_{2} \ldots a_{k-1} a_{k} a_{k+1} \ldots, 0 \overline{1}\right) & =\sum_{i=s+1}^{\infty} \frac{\alpha_{i}^{\prime}+\beta_{i}^{\prime}}{2^{i}} \\
& =\frac{x_{s+1}+1}{2^{s+1}}+\frac{x_{s+2}+1}{2^{s+2}}+\frac{x_{s+3}+1}{2^{s+3}}+\cdots \\
& =\frac{1}{2^{s}}+\frac{x_{s+1}}{2^{s+1}}+\frac{x_{s+2}}{2^{s+2}}+\frac{x_{s+3}}{2^{s+3}}+\cdots
\end{aligned}
$$




$$
\begin{aligned}
d_{1}\left(b_{1} b_{2} \ldots b_{k-1} b_{k} b_{k+1} \cdots, 0 \overline{1}\right)= & \sum_{i=2}^{\infty} \frac{\alpha_{i}^{\prime \prime}+\beta_{i}^{\prime \prime}}{2^{i}} \\
= & \frac{0+0}{2^{2}}+\frac{0+0}{2^{3}}+\ldots+\frac{0+0}{2^{s-1}}+\frac{1+0}{2^{s}} \\
& +\frac{x_{s+1}+0}{2^{s+1}}+\frac{x_{s+2}+0}{2^{s+2}}+\cdots \\
= & \frac{1}{2^{s}}+\frac{x_{s+1}}{2^{s+1}}+\frac{x_{s+2}}{2^{s+2}}+\frac{x_{s+3}}{2^{s+3}}+\cdots
\end{aligned}
$$

where $x_{i} \in\{0,1\}$ for $i=s+1, s+2, s+3, \ldots$ This completes the proof.

In a similar way, we can generalize Proposition 6 as follows:

Corollary 7. Let $a=a_{1} a_{2} \ldots a_{k-1} a_{k} a_{k+1} \ldots$ and $b=b_{1} b_{2} \ldots b_{k-1} b_{k} b_{k+1} \ldots$ be arbitrary elements of $\Sigma_{2} / \sim$. If $a_{i}=b_{i}=\sigma_{i}$ for all $i=1,2, \ldots, k-1, a_{k} \neq b_{k}$ and $\alpha_{i}=\beta_{i}$ for all $i=k+1, k+2, \ldots$, then these points are symmetric with respect to the point $\sigma_{1} \sigma_{2} \ldots \sigma_{k-1} 0 \overline{1}$ (or equivalently $\sigma_{1} \sigma_{2} \ldots \sigma_{k-1} 1 \overline{0}$ ) where $x_{i} \in\{0,1\}$ for $i=1,2, \ldots, k-1$.

Proof. The proof will be omitted.

The following proposition gives us the relationship between truncation errors for the computations which is obtained by the metrics $d_{\text {eucl }}$ and $d_{1}$. In Proposition 8 , we choose $a$ and $b$ as

$$
a_{1} a_{2} \ldots a_{k-1} a_{k} a_{k+1} \ldots a_{n} a_{n+1} a_{n+1} \ldots
$$

and

$$
b_{1} b_{2} \ldots b_{k-1} b_{k} b_{k+1} \ldots b_{n} b_{n+1} b_{n+1} \ldots
$$

respectively where $a_{i}=b_{i}$ for $i=1,2, \ldots k-1$ and $a_{k} \neq b_{k}$.

Proposition 8. The truncation errors $E_{n}$ and $E_{n+1}^{\prime}$ are determined such that

$$
d_{1}(a, b)=\sum_{i=k+1}^{n} \frac{\alpha_{i}+\beta_{i}}{2^{i}}+E_{n}
$$

and

$$
d_{\text {eucl }}\left(h^{\prime}(a), h^{\prime}(b)\right)=\left|\sum_{i=k}^{n} \frac{a_{i}-b_{i}}{2^{i}}+E_{n+1}^{\prime}\right| .
$$

Then we have

$$
E_{n}=2^{-n}+E_{n+1}^{\prime} .
$$


Proof. It must be either $b_{k}>a_{k}$ or $a_{k}>b_{k}$ since $a_{k} \neq b_{k}$. Without loss of generality, let us take $a_{k}>b_{k}$ and then we have $a_{k}=1$ and $b_{k}=0$. With simple calculations, we obtain

$$
\begin{aligned}
& d_{1}(a, b)=\sum_{i=k+1}^{\infty} \frac{\alpha_{i}+\beta_{i}}{2^{i}} \\
& =\sum_{i=k+1}^{n} \frac{\alpha_{i}+\beta_{i}}{2^{i}}+E_{n} \\
& =\frac{\left|a_{k+1}-b_{k}\right|+\left|b_{k+1}-a_{k}\right|}{2^{k+1}}+\frac{\left|a_{k+2}-b_{k}\right|+\left|b_{k+2}-a_{k}\right|}{2^{k+2}} \\
& +\cdots+\frac{\left|a_{n}-b_{k}\right|+\left|b_{n}-a_{k}\right|}{2^{n}}+E_{n} \\
& =\frac{\left(a_{k+1}-b_{k}\right)+\left(a_{k}-b_{k+1}\right)}{2^{k+1}}+\frac{\left(a_{k+2}-b_{k}\right)+\left(a_{k}-b_{k+2}\right)}{2^{k+2}} \\
& +\cdots+\frac{\left(a_{n}-b_{k}\right)+\left(a_{k}-b_{n}\right)}{2^{n}}+E_{n} \\
& =\frac{\left(a_{k}-b_{k}\right)+\left(a_{k+1}-b_{k+1}\right)}{2^{k+1}}+\frac{\left(a_{k}-b_{k}\right)+\left(a_{k+2}-b_{k+2}\right)}{2^{k+2}} \\
& +\cdots+\frac{\left(a_{k}-b_{k}\right)+\left(a_{n}-b_{n}\right)}{2^{n}}+E_{n} \\
& =\left(\frac{1}{2^{k+1}}+\frac{1}{2^{k+2}}+\cdots+\frac{1}{2^{n}}\right)+\frac{\left(a_{k+1}-b_{k+1}\right)}{2^{k+1}}+\frac{\left(a_{k+2}-b_{k+2}\right)}{2^{k+2}} \\
& +\cdots+\frac{\left(a_{n}-b_{n}\right)}{2^{n}}+E_{n} \\
& =\frac{1-2^{k-n}}{2^{k}}+\frac{\left(a_{k+1}-b_{k+1}\right)}{2^{k+1}}+\frac{\left(a_{k+2}-b_{k+2}\right)}{2^{k+2}}+\cdots+\frac{\left(a_{n}-b_{n}\right)}{2^{n}}+E_{n}
\end{aligned}
$$

Moreover, we compute

$$
\begin{aligned}
d_{\text {eucl }}\left(h^{\prime}(a), h^{\prime}(b)\right)= & \left|\sum_{i=k}^{n} \frac{a_{i}-b_{i}}{2^{i}}+E_{n+1}^{\prime}\right| \\
= & \sum_{i=k}^{n} \frac{a_{i}-b_{i}}{2^{i}}+E_{n+1}^{\prime} \\
= & \frac{1}{2^{k}}+\frac{\left(a_{k+1}-b_{k+1}\right)}{2^{k+1}}+\frac{\left(a_{k+2}-b_{k+2}\right)}{2^{k+2}}+\cdots+\frac{\left(a_{n}-b_{n}\right)}{2^{n}} \\
& +E_{n+1}^{\prime}
\end{aligned}
$$


We know that $d_{1}(a, b)=d_{\text {eucl }}\left(h^{\prime}(a), h^{\prime}(b)\right)$ because of Proposition 2 Thus,

$$
E_{n}=2^{-n}+E_{n+1}^{\prime}
$$

is obtained if the required simplifications are made. Observe that, this equation does not depend on the choice of $k$ and the error $E_{n}$ is always positive while the error $E_{n+1}^{\prime}$ can be positive or negative.

As seen in the formula (8), the metric $d_{1}$ works better in some computations. An example which shows that the absolute value of truncation error $E_{n}$ is less than the absolute value of truncation error $E_{n+1}^{\prime}$ is given as follows:

Example 9. Consider the points $a_{1} a_{2} \ldots a_{k-1} 0111 \ldots$ and $a_{1} a_{2} \ldots a_{k-1} 1000 \ldots$ It is obvious that truncation error $E_{n}$ is always zero for every natural number $n$. But, truncation error $E_{n+1}^{\prime}$ equals to $-2^{-n}$.

\section{CONCLUSION}

We give a metric formula on the quotient space $\Sigma_{2} / \sim$. Using a similar method, metric formulas on quotient spaces, which are related to the sequence spaces $\Sigma_{n}$ for every natural $n$, can be defined (for an example see ( [6] ). Moreover, the metric spaces which are isometric to these quotient spaces equipped with these metrics can be investigated. In this paper, we show the metric space $\left(\Sigma_{2} / \sim, d_{1}\right)$ is isometric to the $\left([0,1], d_{\text {eucl }}\right)$. The metric $d_{1}$ provides some facilities since there is no absolute value and subtraction in the metric formula (for details see Example 5, 9 and Proposition 8). The distance is also obtained as a binary number. As the metric formula $d_{1}$ is simple and understandable, it can also be used in the computer science applications (that is, the metric formula (7) is quite suitable for the basic coding commands).

The metric formula $d_{1}$ also has a different interpretation from the Euclidean metric defined on $[0,1]$ : The distance between two points in the Euclidean metric is formulated as the absolute value of the difference of the distances of these two points to the point 0 while the distance between two points in the metric $d_{1}$ is formulated as the sum of the distances of these points to the point $\frac{1}{2^{k}}$ where $k$ is the smallest index such that $a_{i} \neq b_{i}$ for $i=1,2,3, \ldots$ (for details see Proposition 6 and Corollary 7 .

Authors Contribution Statement The authors contributed equally to all parts of the paper.

Declaration of Competing Interest The authors have no potential conflict of interest. 


\section{REFERENCES}

[1] Barnsley, M. F., Fractals Everywhere, Academic Press, Boston, MA, USA, 1988.

[2] Barnsley, M. F., Superfractals, Cambridge University Press, New York, USA, 2006.

[3] Burago, D., Burago, Y., Ivanov, S., A Course in Metric Geometry, AMS, San Diego, CA, USA, 2001.

[4] Falconer, K. J., Fractal Geometry, Mathematical Foundations and Application, John Wiley, UK, 2014.

[5] Hutchinson, J. E., Fractals and self-similarity, Indiana Univ. Math. J., 30 (1981), 713-747, http://dx.doi.org/10.1512/iumj.1981.30.30055.

[6] Saltan, M., Özdemir, Y., Demir, B., An explicit formula of the intrinsic metric on the Sierpinski gasket via code representation, Turkish J. Math., 42(2) (2018), 716-725, http://doi.org/10.3906/mat-1702-55.

[7] Saltan, M., Özdemir, Y., Demir, B., Geodecisc of the Sierpinski gasket, Fractals, 26(3) (2018), 1850024, https://doi.org/10.1142/S0218348X1850024X.

[8] Williams, S. G., Symbolic dynamics and its applications, In Proceedings of Symposia in Applied Mathematics, AMS, 60 (2004), 1-11, https://doi.org/10.1090/psapm/060. 